\title{
Changes in body composition and skeletal robustness in 7-17-year-old children and adolescents from Plovdiv, Bulgaria (1998-2008)
}

\section{DOI: http://doi.org/10.26758/9.1.4}

Mima NIKOLOVA (1), Silviya MLADENOVA (2), Doychin BOYADZHIEV (3), Tanya PASKALEVA (4)

(1) Department of Human Anatomy and Physiology, "Paisii Hilendarski" University at Plovdiv, Plovdiv, Bulgaria

(2) Department of Natural - Mathematical Sciences, "Paisii Hilendarski" University at Plovdiv Smolyan Branch, Smolyan, Bulgaria

(3) Department of Applied Mathematics and Modeling, "Paisii Hilendarski" University at Plovdiv, Plovdiv, Bulgaria

(4) Department of Health Care, Trakiya University - Haskovo Branch, Haskovo, Bulgaria

Address correspondence to: Silviya Mladenova, Department of Natural-Mathematical Sciences, "Paisii Hilendarski" University at Plovdiv - Smolyan Branch, 32, Dicho Petrov, 4700 Smolyan, Bulgaria, E-mail: silviamladenova.sm@gmail.com

\begin{abstract}
Objectives. Overweight and obesity among children and adolescents represent a global epidemic problem that leads to a number of socially serious diseases among grown-ups. The purpose of this study is to assess the changes that have occurred for a 10-year period of time in body composition and skeletal robustness of children and adolescents.

Material and methods. 2094 healthy children and adolescents aged 7-17 from the town of Plovdiv were examined in 2008/2009. The results were compared with identical ones referring to healthy 7-17 year-old children and adolescents from Plovdiv, who were examined in 1998-1999. For each person height, weight, elbow breadth, subscapular and triceps skinfolds were measured using anthropometric methods. On this basis, calculations of the body mass index, the fat free mass index, the fat mass index, percentage of body fat and the Frame- Index of each child were made. The data were processed by SPSS 20.0 software.

Results. The children from this survey are slightly taller and heavier, with the values of BMI and a significantly lower percentage of body fat tending to increase. During the 10 years period of survey, the Frame has decreased significantly in almost all age groups, except 11 and 16-yearold boys and 17-year-old girls from the previous study. Skeletal robustness has not decreased in any percentile groups: the 90 and 97 percentile values are higher among children nowadays, and the values of the $10^{\text {th }}$ and $3^{\text {rd }}$ percentile of the index are lower. The ten-year variations in the values of $10^{\text {th }}$ percentile correspond to lower skeletal robustness from 2 to $15.7 \%$ for boys and from 7 to $18 \%$ for girls. There are no significant correlations between skeletal robustness and BMI, as well as between skeletal robustness and percentage of body fat.

Conclusions. While growing, contemporary children accumulate more fat-free body mass per unit of height, whereas their peers a decade ago - more fat tissue per unit of height. The increase in the percentage of overweight and the decrease in skeletal robustness of contemporary children and adolescents from Plovdiv were caused, entirely or partially, by reduced physical activity.
\end{abstract}

Keywords: body composition, skeletal robustness, changes, school children. 


\section{Introduction}

As a matter of biological importance and from a medical point of view as well, it is essential to study body composition because it will give us an idea of the ratio of the components forming human body weight. The assessment of body composition of children and adolescents is a matter of particular importance, since deterioration in their physical development and reduced health indicators occurred during the last decade. Moreover, there is an increased frequency of occurrence of adolescents with insufficient and excessive body mass.

According to Popkin, Adair and Ng (2012) changes in lifestyle, food systems and dietary habits are consequences. The nutrition transition results in increased energy intake and with the advancement of techniques physical activity decreases. The reduced physical activity and sedentary behaviour of contemporary generation children and adolescents are more frequent (Basterfield et al., 2011; Verloigne et al., 2012). Furthermore, children in more European counties spend more and more time watching TV and participate in sports clubs less frequently, such behaviour leading to lower physical activity (Klimatskaya, Laskiene and Shpakou, 2011; Lömmle et al., 2012). Reduced physical activity is not only one of the reasons for obesity, but also a cause of the impairment of the external skeletal robustness resulting in a decrease of it (Sheffler, 2011; Rietsch, Godina and Scheffler, 2013). On the other hand, an imbalance of energy intake and consumption leads to obesity among children and adults in industrial and developing countries (Popkin, Adair and Ng, 2012). High consumption of sweets, snack-type products, as well as eating in front of the TV, have increased the percentage of overweight among contemporary children, compared to their peers in 1970s according to data found about children in Romania (Baciu, 2011). These disorders occur frequently due to restrictive and dull diets, for such reason being important to ensure adequate nutritional intake, especially for adolescents (Baciu, 2013).

WHO classifies overweight, and especially obesity among adolescents, as a global epidemic problem, causing a predisposition to socially significant diseases among grown-ups (Erbersdobler, Heseker and Wolfram, 2005).

The body mass index (BMI), which describes only the height-weight ratios, is used to determine body nutritional status. However, an individual's developmental status and nutritional evaluation cannot be estimated in a similar way by means of the relation between height and weight only, because while providing a general idea of the total body mass quantitative changes, they do not provide explanation on what expenses those changes are carried out (Chtecov, 1970). Proper development evaluation requires approaching the methods and techniques that reveal an organism's qualitative changes occurring at certain stages of its development. Similar approaches are associated with investigation of the components constituting body mass, as the body composition (Frisancho, 1984; Frisancho, 1990; Mitova, 2009; Mladenova and Nikolova, 2005; Mladenova and Kodgebasheva, 2010; Rolland - Cachera, 1998; Freedman et al., 2005). A number of studies show that high BMI correlates with a higher percentage of body fat (Daniels, Khoury and Morrison, 1997; Deurenberg, Weststrate and Seidell, 1991; Pietrobelli et al., 1998) and other components.

For a growing-up organism, however, it is essential to have detailed information about the development of the components of body mass. Thus, prevention of the decrease can start from early childhood

The assessment of different body components of children and adolescents from Plovdiv and comparing them with the results from the previous study will give more information about the development of body composition and external skeletal robustness in the period between 1998 and 2008. 


\section{Material and methods}

\section{Sample}

A number of 2094 Bulgarian children and adolescents from Plovdiv (1040 boys and 1054 girls), aged 7 to 17 , were cross-sectionally examined in 2008-2009. The comparative sample includes 1913 Bulgarian children and adolescents (1024 boys and 889 girls), who were examined in 1998-1999 by the authors. All children in the two samples of this study were clinically healthy and of Bulgarian nationality and origin. The children with chronicle diseases of the skeletalmuscular system, diabetes type A, hormonal disorders and heredity diseases were excluded from the sample. The children belonging to Roma, Turkish and other ethnical groups were also excluded from the sample.

\section{Ethics statement}

The study was made with the official approval of the Regional Inspectorate of Education in Plovdiv, Bulgaria's Ministry of Education and Science and of the Ethical Committee of Plovdiv University A written informed consent of the parents or guardians of each child included in the research group, was obtained in accordance with the ethical principles for medical research involving human subjects in the Helsinki Declaration of World Medical Association (World Medical Association Declaration of Helsinki, 2000). Measurements were taken in elementary and secondary schools in the town of Plovdiv (Bulgaria). The schools are in different regions of the town therefore children belonging to them vary in social backgrounds.

\section{Anthropometric measurements}

For each person height, weight, elbow breadth, subscapular and triceps skinfolds were taken using anthropometric methods. Anthropometric measurements were followed by the standardized methods of Martin and Saller (1959) in a standing position. The measurements were taken with prescribed measuring anthropometric instruments.

By means of such method, the following indices were calculated and compared: the percentage of Body Fat (\% BF), Fat Mass (FM, kg), Fat-Free Mass (FFM), Body Mass Index $\left(\mathrm{BMI}, \mathrm{kg} / \mathrm{m}^{2}\right)$, Fat Mass Index $\left(\mathrm{FMI}, \mathrm{kg} / \mathrm{m}^{2}\right)$ and Fat- Free Mass Index (FFMI, $\mathrm{kg} / \mathrm{m}^{2}$ ) and Frame-Index (FrI). The percentages of Body Fat (\% BF), Fat Mass (FM, kg) and Fat - Free Mass (FFM, kg) were calculated by means of the regression equations of Slaughter et al. (1988). Fat Mass Index (FMI) and Fat-Free Mass Index (FFMI) were defined according to Freedman et al. (2005). Frame-Index was calculated by Frisancho's formulae (1990):

Frame Index $=($ elbow breadth $(\mathrm{mm}) \times 100) /$ height $(\mathrm{cm})$

\section{Statistical analysis}

The descriptive statistics, the correlation and the non-parametric analysis were used. Mean and standard deviation and the $10^{\text {th }}, 50^{\text {th }}$ and $90^{\text {th }}$ percentiles for each of the anthropometric measurements and indexes were calculated. In addition, the $3^{\text {rd }}$ and $97^{\text {th }}$ percentiles for the Frame index were calculated. The correlation coefficients between the Frame-Index, the Body Mass Index and the Body Fat $(\% \mathrm{BF})$ percentage for each age and gender group were calculated. The data were processed by SPSS 20.0 software. The statistical significance of the two samples was evaluated through T-test of Student on $\mathrm{p}$-level $\leq 0.05$. 


\section{Results}

Table 1 presents the results of the mean(Mean),the standard deviation (SD) and percentiles $10^{\text {th }}, 50^{\text {th }}$ and $90^{\text {th }}$ of the height, weight, $\%$ body fat, the body mass index, the fat mass index, the fat-free mass index and the Frame index, according to the age and sex of the girls from the samples of the years 1998 and 2008.

Table 1. Basic statistical characteristics of anthropometric indicators of the girls

\begin{tabular}{|c|c|c|c|c|c|c|c|c|c|c|c|c|}
\hline \multirow[b]{2}{*}{$\begin{array}{l}\text { Age } \\
\text { (y) }\end{array}$} & \multirow{2}{*}{$\begin{array}{l}\text { GIRLS } \\
\text { Indicators } \\
\end{array}$} & \multicolumn{5}{|c|}{1998} & \multicolumn{5}{|c|}{2008} & \multirow{2}{*}{$\begin{array}{c}9 / 9 \\
p \\
\end{array}$} \\
\hline & & Mean & SD & $\mathbf{P}_{10}$ & $\mathbf{P}_{50}$ & $P_{90}$ & Mean & SD & $P_{10}$ & $\mathbf{P}_{50}$ & $\mathbf{P}_{90}$ & \\
\hline \multirow{7}{*}{7} & Height, cm & 125.9 & 5.6 & 117.7 & 126.6 & 132.0 & 127.2 & 5.6 & 121.0 & 127.0 & 135.0 & .164 \\
\hline & Weight, kg & 26.4 & 5.3 & 21.0 & 25.2 & 35.2 & 26.6 & 5.1 & 20.4 & 25.6 & 33.4 & .841 \\
\hline & BMI, $\mathrm{kg} / \mathrm{m}^{2}$ & 16.7 & 3.1 & 13.8 & 15.7 & 22.5 & 16.4 & 2.5 & 13.6 & 15.8 & 19.6 & .517 \\
\hline & FMI, kg/m² & 3.65 & 1.53 & 2.21 & 3.03 & 6.17 & 2.86 & 1.53 & 1.42 & 2.36 & 4.94 & .016 \\
\hline & FFMI, $\mathrm{kg} / \mathrm{m}^{2}$ & 13.03 & 1.91 & 11.08 & 12.35 & 15.92 & 13.50 & 1.39 & 11.94 & 13.49 & 15.02 & .214 \\
\hline & $\% \mathrm{BF}$ & 21.16 & 4.53 & 15.87 & 20.95 & 27.99 & 16.78 & 6.17 & 9.93 & 14.71 & 26.17 & .000 \\
\hline & FrI & 44.00 & 3.94 & 39.23 & 43.38 & 49.19 & 37.75 & 3.81 & 33.23 & 37.50 & 42.46 & .000 \\
\hline \multirow{7}{*}{8} & Height, $\mathrm{cm}$ & 129.1 & 5.8 & 120.4 & 129.6 & 137.7 & 131.9 & 6.5 & 124.0 & 131.7 & 139.1 & .006 \\
\hline & Weight, kg & 29.4 & 6.1 & 22.0 & 27.6 & 38.9 & 30.3 & 7.4 & 22.8 & 28.5 & 42.8 & .463 \\
\hline & $\mathrm{BMI}, \mathrm{kg} / \mathrm{m}^{2}$ & 17.6 & 2.8 & 14.5 & 17.0 & 21.4 & 17.2 & 3.0 & 14.2 & 16.4 & 21.9 & .482 \\
\hline & FMI, $\mathrm{kg} / \mathrm{m}^{2}$ & 3.88 & 1.58 & 2.22 & 3.48 & 6.32 & 3.12 & 1.83 & 1.57 & 2.43 & 5.53 & .014 \\
\hline & FFMI, $\mathrm{kg} / \mathrm{m}^{2}$ & 13.90 & 1.56 & 11.97 & 13.79 & 16.28 & 14.11 & 1.50 & 12.33 & 13.95 & 16.36 & .427 \\
\hline & $\% \mathrm{BF}$ & 21.16 & 5.62 & 14.01 & 20.39 & 29.57 & 17.13 & 6.61 & 10.56 & 15.08 & 26.77 & .000 \\
\hline & FrI & 40.46 & 3.73 & 37.00 & 40.09 & 43.73 & 38.86 & 3.79 & 34.74 & 38.32 & 44.63 & .010 \\
\hline \multirow{7}{*}{9} & Height, cm & 133.9 & 5.5 & 127.2 & 133.8 & 141.1 & 137.0 & 7.2 & 128.0 & 137.0 & 146.0 & .001 \\
\hline & Weight, kg & 31.6 & 6.1 & 24.5 & 30.6 & 38.8 & 33.9 & 8.7 & 24.7 & 32.0 & 47.1 & .030 \\
\hline & BMI, $\mathrm{kg} / \mathrm{m}^{2}$ & 17.5 & 2.7 & 14.3 & 17.5 & 21.2 & 17.9 & 3.4 & 14.4 & 16.9 & 22.0 & .448 \\
\hline & FMI, kg/m² & 3.75 & 1.35 & 2.35 & 3.49 & 5.87 & 3.61 & 1.99 & 1.73 & 2.96 & 6.10 & .581 \\
\hline & FFMI, $\mathrm{kg} / \mathrm{m}^{2}$ & 13.69 & 1.55 & 11.77 & 13.54 & 15.59 & 14.27 & 1.66 & 12.35 & 13.85 & 16.46 & .021 \\
\hline & $\% \mathrm{BF}$ & 20.97 & 4.92 & 14.94 & 21.21 & 27.85 & 19.15 & 6.60 & 11.59 & 17.31 & 28.07 & .036 \\
\hline & FrI & 40.86 & 3.52 & 37.55 & 40.33 & 44.64 & 39.41 & 3.89 & 34.43 & 39.11 & 44.79 & .006 \\
\hline \multirow{7}{*}{10} & Height, $\mathrm{cm}$ & 140.8 & 7.7 & 130.5 & 141.5 & 149.3 & 144.2 & 7.5 & 133.8 & 144.0 & 155.0 & .003 \\
\hline & Weight, $\mathrm{kg}$ & 37.2 & 9.3 & 26.4 & 35.5 & 50.7 & 39.2 & 9.3 & 28.0 & 38.5 & 53.8 & .137 \\
\hline & $\mathrm{BMI}, \mathrm{kg} / \mathrm{m}^{2}$ & 18.5 & 3.4 & 14.8 & 18.0 & 24.1 & 18.7 & 3.6 & 14.5 & 18.1 & 23.4 & .708 \\
\hline & FMI, $\mathrm{kg} / \mathrm{m}^{2}$ & 4.43 & 2.20 & 2.17 & 3.88 & 7.84 & 3.70 & 1.83 & 1.83 & 3.11 & 6.55 & .020 \\
\hline & FFMI, $\mathrm{kg} / \mathrm{m}^{2}$ & 14.29 & 1.70 & 12.28 & 14.07 & 16.83 & 15.04 & 2.22 & 12.60 & 14.48 & 17.84 & .014 \\
\hline & $\% \mathrm{BF}$ & 22.51 & 7.12 & 13.49 & 21.50 & 33.83 & 18.88 & 6.48 & 12.07 & 17.23 & 28.21 & .001 \\
\hline & FrI & 40.74 & 2.96 & 37.55 & 41.17 & 44.38 & 41.82 & 5.73 & 34.86 & 40.94 & 48.76 & .118 \\
\hline \multirow{7}{*}{11} & Height, cm & 147.8 & 8.2 & 137.8 & 147.3 & 156.4 & 147.4 & 8.6 & 135.2 & 149.0 & 160.0 & .793 \\
\hline & Weight, kg & 40.9 & 9.7 & 28.9 & 40.0 & 54.5 & 40.4 & 9.6 & 28.3 & 39.5 & 55.7 & .746 \\
\hline & BMI, $\mathrm{kg} / \mathrm{m}^{2}$ & 18.5 & 3.3 & 14.6 & 18.2 & 23.5 & 18.4 & 3.3 & 14.7 & 17.8 & 23.1 & .799 \\
\hline & FMI, kg/m² & 4.28 & 2.03 & 2.14 & 3.72 & 7.25 & 3.27 & 1.61 & 1.56 & 2.96 & 5.47 & .000 \\
\hline & FFMI, $\mathrm{kg} / \mathrm{m}^{2}$ & 14.16 & 1.64 & 12.32 & 14.07 & 16.30 & 15.15 & 2.16 & 12.72 & 14.81 & 17.70 & .001 \\
\hline & $\% \mathrm{BF}$ & 22.29 & 6.86 & 13.96 & 20.77 & 33.35 & 17.10 & 5.81 & 9.76 & 16.71 & 25.63 & .000 \\
\hline & FrI & 39.97 & 2.37 & 37.46 & 39.83 & 43.23 & 40.06 & 4.61 & 34.95 & 38.69 & 47.78 & .876 \\
\hline \multirow{7}{*}{12} & Height, $\mathrm{cm}$ & 153.3 & 7.2 & 144.4 & 154.0 & 161.6 & 154.5 & 7.8 & 142.2 & 155.0 & 164.0 & .263 \\
\hline & Weight, kg & 45.5 & 10.4 & 34.4 & 43.4 & 59.5 & 48.3 & 12.2 & 34.5 & 46.0 & 61.9 & .101 \\
\hline & BMI, $\mathrm{kg} / \mathrm{m}^{2}$ & 19.3 & 3.6 & 15.3 & 18.5 & 25.2 & 20.0 & 3.9 & 15.5 & 19.4 & 24.7 & .170 \\
\hline & FMI, $\mathrm{kg} / \mathrm{m}^{2}$ & 4.41 & 2.05 & 2.49 & 3.74 & 7.38 & 4.10 & 1.97 & 1.99 & 3.60 & 6.85 & .296 \\
\hline & FFMI, $\mathrm{kg} / \mathrm{m}^{2}$ & 14.86 & 1.91 & 12.52 & 14.87 & 17.27 & 15.92 & 2.51 & 13.12 & 15.65 & 19.62 & .002 \\
\hline & $\% \mathrm{BF}$ & 22.00 & 6.03 & 15.45 & 20.92 & 31.39 & 19.75 & 6.40 & 11.59 & 18.24 & 29.25 & .014 \\
\hline & FrI & 39.42 & 2.68 & 35.88 & 39.38 & 43.00 & 39.40 & 12.37 & 32.90 & 37.12 & 44.45 & .986 \\
\hline \multirow{7}{*}{13} & Height, cm & 158.8 & 5.8 & 151.9 & 158.6 & 166.5 & 159.2 & 5.6 & 152.0 & 159.0 & 167.0 & .559 \\
\hline & Weight, kg & 51.5 & 11.8 & 39.2 & 50.0 & 66.6 & 53.8 & 12.2 & 39.2 & 52.0 & 72.1 & .186 \\
\hline & BMI, $\mathrm{kg} / \mathrm{m}^{2}$ & 20.4 & 4.3 & 15.8 & 19.3 & 26.4 & 21.1 & 4.3 & 16.4 & 20.2 & 26.9 & .221 \\
\hline & FMI, $\mathrm{kg} / \mathrm{m}^{2}$ & 5.22 & 2.81 & 2.39 & 4.42 & 9.03 & 4.45 & 2.24 & 2.39 & 3.93 & 7.72 & .035 \\
\hline & FFMI, $\mathrm{kg} / \mathrm{m}^{2}$ & 15.42 & 2.02 & 13.24 & 14.93 & 18.08 & 16.68 & 3.10 & 13.77 & 15.94 & 21.06 & .001 \\
\hline & $\% \mathrm{BF}$ & 23.97 & 7.62 & 15.19 & 23.42 & 35.80 & 20.43 & 7.07 & 12.75 & 19.06 & 31.21 & .001 \\
\hline & FrI & 39.12 & 3.46 & 35.36 & 38.91 & 42.12 & 39.21 & 6.85 & 33.09 & 36.65 & 50.13 & .907 \\
\hline \multirow{7}{*}{14} & Height, cm & 161.5 & 5.5 & 155.0 & 161.6 & 168.5 & 160.3 & 5.4 & 152.0 & 160.0 & 168.0 & .131 \\
\hline & Weight, kg & 55.3 & 12.5 & 43.2 & 52.2 & 71.3 & 54.6 & 10.1 & 44.5 & 52.5 & 69.4 & .642 \\
\hline & BMI, $\mathrm{kg} / \mathrm{m}^{2}$ & 21.1 & 4.3 & 16.9 & 20.1 & 26.3 & 21.2 & 3.7 & 16.9 & 20.5 & 26.0 & .894 \\
\hline & FMI, $\mathrm{kg} / \mathrm{m}^{2}$ & 5.86 & 2.70 & 3.18 & 5.10 & 10.05 & 4.92 & 2.20 & 2.61 & 4.25 & 8.08 & .013 \\
\hline & FFMI, $\mathrm{kg} / \mathrm{m}^{2}$ & 15.88 & 2.15 & 13.52 & 15.47 & 17.98 & 16.31 & 2.10 & 13.79 & 16.23 & 18.74 & .196 \\
\hline & $\% \mathrm{BF}$ & 25.85 & 6.46 & 18.74 & 24.90 & 35.21 & 22.41 & 6.58 & 14.60 & 21.50 & 31.39 & .001 \\
\hline & FrI & 39.07 & 2.91 & 35.78 & 38.96 & 42.14 & 38.31 & 8.44 & 31.50 & 36.02 & 46.52 & .389 \\
\hline 15 & Height, cm & 161.6 & 5.5 & 154.3 & 161.5 & 169.8 & 161.0 & 5.8 & 153.0 & 161.5 & 169.0 & .533 \\
\hline 15 & Weight, kg & 53.2 & 10.1 & 41.3 & 52.5 & 65.9 & 55.1 & 8.6 & 45.0 & 54.3 & 67.7 & .172 \\
\hline
\end{tabular}




\begin{tabular}{|c|c|c|c|c|c|c|c|c|c|c|c|c|}
\hline \multirow[b]{2}{*}{$\begin{array}{l}\text { Age } \\
\text { (y) }\end{array}$} & \multirow{2}{*}{$\begin{array}{l}\text { GIRLS } \\
\text { Indicators }\end{array}$} & \multicolumn{5}{|c|}{1998} & \multicolumn{5}{|c|}{2008} & \multirow{2}{*}{$\begin{array}{c}/ 9 \\
p\end{array}$} \\
\hline & & Mean & SD & $\mathbf{P}_{10}$ & $\mathbf{P}_{50}$ & $\mathbf{P}_{90}$ & Mean & SD & $\mathbf{P}_{10}$ & $\mathbf{P}_{50}$ & $\mathbf{P}_{90}$ & \\
\hline & $\mathrm{BMI}, \mathrm{kg} / \mathrm{m}^{2}$ & 20.4 & 3.7 & 16.5 & 19.7 & 24.7 & 21.2 & 3.0 & 17.8 & 20.8 & 25.7 & .085 \\
\hline & FMI, $\mathrm{kg} / \mathrm{m}^{2}$ & 5.73 & 2.45 & 2.97 & 5.24 & 8.65 & 4.66 & 1.73 & 2.79 & 4.34 & 6.93 & .015 \\
\hline & FFMI, $\mathrm{kg} / \mathrm{m}^{2}$ & 15.68 & 2.49 & 13.13 & 15.57 & 18.65 & 16.58 & 1.97 & 14.40 & 16.13 & 19.41 & .026 \\
\hline & $\% \mathrm{BF}$ & 25.92 & 6.66 & 17.40 & 25.02 & 34.16 & 21.52 & 5.79 & 14.71 & 20.92 & 29.52 & .000 \\
\hline & FrI & 37.81 & 2.75 & 34.61 & 37.61 & 41.05 & 37.63 & 5.69 & 31.99 & 36.07 & 47.01 & .785 \\
\hline \multirow{7}{*}{16} & Height, $\mathrm{cm}$ & 162.5 & 6.1 & 153.9 & 162.1 & 169.6 & 162.8 & 5.9 & 156.0 & 162.0 & 170.9 & .729 \\
\hline & Weight, kg & 56.4 & 11.1 & 46.7 & 53.6 & 69.5 & 55.7 & 9.8 & 45.1 & 52.8 & 71.0 & .656 \\
\hline & $\mathrm{BMI}, \mathrm{kg} / \mathrm{m}^{2}$ & 21.4 & 3.9 & 17.8 & 20.3 & 26.8 & 21.0 & 3.2 & 17.3 & 20.1 & 25.8 & .489 \\
\hline & FMI, $\mathrm{kg} / \mathrm{m}^{2}$ & 5.85 & 2.62 & 3.62 & 5.04 & 10.51 & 4.80 & 1.87 & 2.85 & 4.32 & 7.17 & .022 \\
\hline & FFMI, $\mathrm{kg} / \mathrm{m}^{2}$ & 16.18 & 2.08 & 14.08 & 15.65 & 18.95 & 16.15 & 1.88 & 13.80 & 15.92 & 19.04 & .938 \\
\hline & $\% \mathrm{BF}$ & 25.55 & 6.40 & 18.98 & 24.18 & 35.50 & 22.36 & 5.53 & 15.82 & 21.21 & 31.17 & .004 \\
\hline & FrI & 38.04 & 2.55 & 34.59 & 38.01 & 41.57 & 35.78 & 3.83 & 31.65 & 35.58 & 40.38 & .000 \\
\hline \multirow{7}{*}{17} & Height, $\mathrm{cm}$ & 162.6 & 5.7 & 156.0 & 162.1 & 170.8 & 161.1 & 6.4 & 152.4 & 162.0 & 169.6 & .134 \\
\hline & Weight, kg & 54.7 & 8.9 & 46.0 & 53.5 & 62.6 & 54.9 & 9.3 & 43.3 & 54.2 & 66.1 & .888 \\
\hline & $\mathrm{BMI}, \mathrm{kg} / \mathrm{m}^{2}$ & 20.6 & 3.1 & 17.9 & 20.0 & 23.8 & 21.1 & 3.3 & 17.3 & 20.7 & 25.2 & .359 \\
\hline & FMI, $\mathrm{kg} / \mathrm{m}^{2}$ & 5.29 & 2.45 & 3.40 & 4.86 & 7.24 & 3.87 & 1.58 & 2.11 & 3.66 & 5.94 & .000 \\
\hline & FFMI, $\mathrm{kg} / \mathrm{m}^{2}$ & 15.51 & 1.39 & 13.68 & 15.39 & 17.11 & 17.25 & 2.59 & 14.14 & 16.68 & 20.69 & .000 \\
\hline & $\% \mathrm{BF}$ & 24.61 & 6.41 & 17.69 & 23.74 & 31.38 & 18.07 & 5.71 & 10.56 & 17.57 & 24.74 & .000 \\
\hline & FrI & 38.59 & 2.53 & 35.40 & 38.65 & 41.12 & 44.04 & 10.46 & 32.46 & 42.35 & 58.47 & .000 \\
\hline
\end{tabular}

Note: Mean - average mean values; SD - standard deviation; P10 -10 ${ }^{\text {th }}$ percentile; P50 - $50^{\text {th }}$ percentile; P90 - $90^{\text {th }}$ percentile; BMI-Body Mass Index; FMI-Fat Mass Index; FFMI-Fat-Free Mass Index; \% BF-percentage of Body Fat; FrI-Frame-Index; $p$ - level of significance

The average height of 7-year-old girls is $127.2 \pm 5.6 \mathrm{~cm}$ and of 17 -year-olds $-161.1 \pm 6.4$ $\mathrm{cm}$ respectively. The average weight of contemporary 7 -year-old girls is $26.6 \pm 5.1 \mathrm{~kg}$ and $54.9 \pm 9.3 \mathrm{~kg}$ for 17 -year-olds.

The average values of BMI of contemporary girls vary from $16.4 \pm 2.5 \mathrm{~kg} / \mathrm{m}^{2}$ for 7 yearolds to $21.1 \pm 3.3 \mathrm{~kg} / \mathrm{m}^{2}$ for 17 year-olds.. As far as girls are concerned, the differences in average values of BMI between the girls from the two samples are insignificant ( $p>0.05$ ).

The results from the age dynamics of the two components of BMI, such as the fat mass index (FMI) and the fat-free mass index (FFMI) show that the average values of FMI for girls from the sample of 2008 increase by $2.86 \pm 1.53 \mathrm{~kg} / \mathrm{m}^{2}$ for 7 -year-olds to $4.92 \pm 2.20 \mathrm{~kg} / \mathrm{m} 2$ for 14 year-olds. Above this age the values of FMI decrease to $3.87 \pm 1.58 \mathrm{~kg} / \mathrm{m}^{2}$ for 17 -year-olds. The differences between FMI of girls from the two samples (1998 and 2008) are significant during all age periods with the exception of 9 and 12-year-olds. This results show that the average values of fat mass normalized by square meter of height for contemporary girls are significantly lower, compared with the values of their coevals in $1998(\mathrm{p}<0.05)$.

The values of the Fat-Free Mass Index (FFMI) in contemporary girls increase from the average $13.5 \mathrm{~kg} / \mathrm{m}^{2} \pm 1.39 \mathrm{~kg} / \mathrm{m}^{2}$ for 7 year-olds to $17.25 \pm 2.59 \mathrm{~kg} / \mathrm{m}^{2}$ for 17 year-olds, i.e. with an average of $3.75 \mathrm{~kg} / \mathrm{m}^{2}$. During the whole period investigated contemporary girls have significantly higher quantity of FFMI, compared with their coevals in 1998 ( $p<0.05)$.

The other important indicator related to the components of body composition is the percentage of body fat $(\% \mathrm{BF})$. As can be seen in Table 1 , the average body fat $(\% \mathrm{BF})$ percentage increases from $16.78 \% \pm 6.17 \%$ for 7 -year-old girls to $22.4 \% \pm 6.58 \%$ for 14 - year-olds, meaning during puberty. Above the age of 14 the percentage BF decreases to $18.07 \% \pm 5.71 \%$ for 17-year-olds. Contemporary girls have significantly lower \% BF than girls in 1998 during the whole period between 7 and 17 years old $(p<0.05)$. This fact reflected on the values of the Fat Mass Index (FMI).

The Frame-Index is the other important health indicator and the component of body composition. By means of this index we can draw our conclusions about external skeletal robustness. Our results show that the average value of this index is $37.75 \pm 3.81$ index unit (UI) in 7-year-old girls sample and it increases to $44.04 \pm 10.46 \mathrm{UI}$ at the end of the period. The results also show that the values of this index are lower for girls in 2008, especially up to 10 years of age. After that, the differences become fewer and the values of the Frame Index are very similar, 
with the exception of girls of 17 years old, where they significantly increase as far as contemporary girls are concerned.

Table 2 presents the results of the mean (Mean),the standard deviation (SD) and percentiles $10^{\text {th }}, 50^{\text {th }}$ and $90^{\text {th }}$ of height, weight, $\%$ body fat, the body mass index, the fat mass index, the fat-free mass index and the Frame index, according to age and sex for the boys from the samples of the years 1998 and 2008.

Table 2. Basic statistical characteristics of anthropometric indicators of boys

\begin{tabular}{|c|c|c|c|c|c|c|c|c|c|c|c|c|}
\hline \multirow[b]{2}{*}{$\begin{array}{l}\text { Age } \\
\text { (y) }\end{array}$} & \multirow{2}{*}{$\begin{array}{l}\text { BOYS } \\
\text { Indicators }\end{array}$} & \multicolumn{5}{|c|}{1998} & \multicolumn{5}{|c|}{2008} & \multirow{2}{*}{$\frac{\hat{\partial} / \overbrace{}^{\pi}}{\mathbf{p}}$} \\
\hline & & Mean & SD & $\mathbf{P}_{10}$ & $\mathbf{P}_{50}$ & $\mathbf{P}_{90}$ & Mean & SD & $\mathbf{P}_{10}$ & $\mathbf{P}_{50}$ & $\mathbf{P}_{90}$ & \\
\hline \multirow{7}{*}{7} & Height, $\mathrm{cm}$ & 126.7 & 5.3 & 120.2 & 126.5 & 134.5 & 128.0 & 5.8 & 121.0 & 127.0 & 134.4 & .162 \\
\hline & Weight, kg & 26.7 & 4.8 & 22.0 & 26.0 & 32.4 & 28.4 & 6.3 & 21.7 & 27.1 & 35.5 & .073 \\
\hline & $\mathrm{BMI}, \mathrm{kg} / \mathrm{m}^{2}$ & 16.6 & 2.2 & 14.2 & 16.1 & 19.8 & 17.2 & 2.7 & 14.2 & 16.7 & 21.1 & .141 \\
\hline & FMI, $\mathrm{kg} / \mathrm{m}^{2}$ & 3.39 & 1.62 & 1.69 & 2.92 & 6.31 & 2.86 & 1.65 & 1.30 & 2.34 & 5.81 & .184 \\
\hline & FFMI, $\mathrm{kg} / \mathrm{m}^{2}$ & 13.73 & 1.71 & 11.52 & 13.78 & 16.65 & 14.32 & 1.57 & 12.46 & 14.23 & 16.41 & .132 \\
\hline & $\% \mathrm{BF}$ & 19.06 & 5.98 & 11.56 & 17.58 & 29.62 & 15.86 & 6.74 & 8.67 & 13.47 & 26.27 & .039 \\
\hline & FrI & 45.08 & 4.56 & 40.28 & 44.28 & 50.19 & 39.39 & 3.65 & 34.80 & 39.37 & 43.45 & .000 \\
\hline \multirow{7}{*}{8} & Height, $\mathrm{cm}$ & 130.7 & 6.6 & 121.6 & 129.4 & 139.2 & 132.6 & 7.0 & 124.0 & 132.1 & 142.0 & .091 \\
\hline & Weight, kg & 31.8 & 7.9 & 22.6 & 30.0 & 43.2 & 31.8 & 8.4 & 22.5 & 29.8 & 44.0 & .976 \\
\hline & $\mathrm{BMI}, \mathrm{kg} / \mathrm{m}^{2}$ & 18.4 & 3.3 & 14.3 & 18.1 & 23.6 & 17.9 & 3.5 & 14.5 & 17.0 & 22.6 & .299 \\
\hline & FMI, $\mathrm{kg} / \mathrm{m}^{2}$ & 4.01 & 1.93 & 1.92 & 3.57 & 7.11 & 3.31 & 2.19 & 1.41 & 2.49 & 6.37 & .043 \\
\hline & FFMI, $\mathrm{kg} / \mathrm{m}^{2}$ & 14.43 & 1.66 & 12.39 & 14.13 & 17.08 & 14.55 & 1.58 & 12.86 & 14.29 & 16.82 & .642 \\
\hline & $\% \mathrm{BF}$ & 20.80 & 6.70 & 13.10 & 19.83 & 30.42 & 17.24 & 7.73 & 9.41 & 15.13 & 30.04 & .004 \\
\hline & FrI & 41.76 & 2.68 & 38.35 & 42.12 & 44.73 & 40.01 & 4.00 & 35.82 & 39.55 & 45.50 & .001 \\
\hline \multirow{7}{*}{9} & Height, $\mathrm{cm}$ & 136.0 & 6.6 & 127.4 & 135.8 & 144.5 & 137.1 & 8.2 & 129.0 & 136.6 & 145.0 & .294 \\
\hline & Weight, kg & 32.2 & 6.8 & 24.6 & 31.4 & 40.7 & 34.3 & 9.3 & 25.0 & 32.2 & 47.1 & .078 \\
\hline & BMI, $\mathrm{kg} / \mathrm{m}^{2}$ & 17.3 & 3.0 & 14.4 & 16.6 & 21.3 & 18.0 & 3.3 & 14.5 & 17.2 & 24.1 & .111 \\
\hline & FMI, $\mathrm{kg} / \mathrm{m}^{2}$ & 3.64 & 2.10 & 1.74 & 2.96 & 6.45 & 3.46 & 2.37 & 1.40 & 2.73 & 6.93 & .623 \\
\hline & FFMI, $\mathrm{kg} / \mathrm{m}^{2}$ & 14.11 & 1.36 & 12.35 & 13.94 & 16.24 & 14.58 & 1.74 & 12.66 & 14.39 & 16.46 & .072 \\
\hline & $\% \mathrm{BF}$ & 19.46 & 7.37 & 11.67 & 16.93 & 29.90 & 17.96 & 8.47 & 9.77 & 15.61 & 30.61 & .249 \\
\hline & FrI & 41.52 & 2.77 & 37.73 & 41.38 & 45.07 & 40.24 & 3.90 & 35.29 & 40.00 & 45.20 & .007 \\
\hline \multirow{7}{*}{10} & Height, $\mathrm{cm}$ & 141.8 & 6.3 & 133.7 & 141.3 & 149.7 & 143.0 & 6.4 & 135.0 & 142.0 & 151.0 & .240 \\
\hline & Weight, kg & 37.6 & 9.0 & 27.2 & 35.5 & 49.5 & 39.9 & 10.0 & 29.0 & 37.5 & 56.0 & .119 \\
\hline & $\mathrm{BMI}, \mathrm{kg} / \mathrm{m}^{2}$ & 18.5 & 3.4 & 15.1 & 17.9 & 23.7 & 19.3 & 3.7 & 15.3 & 18.3 & 24.5 & .135 \\
\hline & FMI, $\mathrm{kg} / \mathrm{m}^{2}$ & 4.47 & 2.44 & 1.98 & 3.57 & 8.12 & 4.00 & 2.36 & 1.79 & 3.14 & 7.38 & .240 \\
\hline & FFMI, $\mathrm{kg} / \mathrm{m}^{2}$ & 14.39 & 1.39 & 12.71 & 14.29 & 16.46 & 15.31 & 2.10 & 13.19 & 14.87 & 17.55 & .003 \\
\hline & $\% \mathrm{BF}$ & 22.35 & 7.79 & 13.65 & 20.88 & 34.20 & 19.64 & 8.07 & 11.26 & 17.12 & 31.67 & .041 \\
\hline & FrI & 41.84 & 4.09 & 37.60 & 41.46 & 45.78 & 41.93 & 4.33 & 35.66 & 41.79 & 47.33 & .890 \\
\hline \multirow{7}{*}{11} & Height, $\mathrm{cm}$ & 145.6 & 6.1 & 137.5 & 145.6 & 153.1 & 148.6 & 8.1 & 138.0 & 148.0 & 158.4 & .003 \\
\hline & Weight, kg & 38.9 & 9.0 & 30.3 & 37.2 & 49.8 & 44.5 & 13.3 & 30.1 & 41.0 & 63.9 & .000 \\
\hline & $\mathrm{BMI}, \mathrm{kg} / \mathrm{m}^{2}$ & 18.2 & 3.3 & 15.0 & 17.5 & 22.8 & 19.9 & 4.6 & 15.0 & 19.0 & 26.6 & .003 \\
\hline & FMI, $\mathrm{kg} / \mathrm{m}^{2}$ & 4.08 & 2.46 & 1.76 & 3.60 & 7.48 & 4.02 & 3.12 & 1.63 & 2.89 & 7.50 & .891 \\
\hline & FFMI, $\mathrm{kg} / \mathrm{m}^{2}$ & 14.38 & 1.59 & 12.56 & 14.19 & 16.56 & 15.88 & 2.69 & 13.02 & 15.46 & 19.39 & .000 \\
\hline & $\% \mathrm{BF}$ & 20.76 & 7.90 & 11.67 & 20.01 & 32.23 & 18.63 & 9.31 & 10.14 & 15.04 & 29.76 & .121 \\
\hline & FrI & 40.64 & 2.60 & 37.85 & 40.29 & 44.26 & 44.33 & 7.52 & 37.09 & 42.07 & 56.40 & .000 \\
\hline \multirow{4}{*}{12} & Height, $\mathrm{cm}$ & 150.9 & 7.7 & 141.4 & 150.7 & 160.5 & 154.3 & 8.0 & 145.0 & 154.0 & 166.0 & .004 \\
\hline & Weight, kg & 45.5 & 12.1 & 32.3 & 43.6 & 61.8 & 48.2 & 11.9 & 34.0 & 45.8 & 66.5 & .131 \\
\hline & $\mathrm{BMI}, \mathrm{kg} / \mathrm{m}^{2}$ & 19.8 & 4.0 & 15.5 & 18.8 & 25.2 & 20.0 & 3.7 & 15.4 & 19.4 & 26.0 & .651 \\
\hline & FMI, $\mathrm{kg} / \mathrm{m}^{2}$ & 4.76 & 2.96 & 1.68 & 4.26 & 8.56 & 3.25 & 2.12 & 1.29 & 2.66 & 6.29 & .000 \\
\hline
\end{tabular}




\begin{tabular}{|c|c|c|c|c|c|c|c|c|c|c|c|c|}
\hline \multirow[b]{2}{*}{$\begin{array}{l}\text { Age } \\
\text { (y) }\end{array}$} & \multirow{2}{*}{$\begin{array}{l}\text { BOYS } \\
\text { Indicators }\end{array}$} & \multicolumn{5}{|c|}{1998} & \multicolumn{5}{|c|}{2008} & \multirow{2}{*}{$\frac{\delta / \overbrace{}^{\Uparrow}}{\mathbf{p}}$} \\
\hline & & Mean & SD & $\mathbf{P}_{10}$ & $\mathbf{P}_{50}$ & $\mathbf{P}_{90}$ & Mean & SD & $\mathbf{P}_{10}$ & $\mathbf{P}_{50}$ & $\mathbf{P}_{90}$ & \\
\hline & FFMI, $\mathrm{kg} / \mathrm{m}^{2}$ & 15.67 & 1.78 & 13.84 & 15.28 & 17.97 & 16.77 & 2.55 & 14.03 & 16.53 & 20.39 & .001 \\
\hline & $\% \mathrm{BF}$ & 21.68 & 9.03 & 10.81 & 21.03 & 34.74 & 15.38 & 7.58 & 7.77 & 12.95 & 26.16 & .000 \\
\hline & FrI & 41.35 & 3.51 & 37.82 & 41.40 & 45.29 & 40.47 & 6.39 & 34.75 & 39.51 & 46.65 & .243 \\
\hline \multirow{7}{*}{13} & Height, $\mathrm{cm}$ & 160.8 & 8.3 & 150.2 & 160.2 & 172.0 & 160.7 & 8.2 & 151.0 & 160.0 & 172.0 & .959 \\
\hline & Weight, kg & 54.1 & 14.0 & 38.1 & 51.6 & 72.9 & 54.6 & 13.3 & 38.7 & 52.7 & 72.0 & .798 \\
\hline & BMI, $\mathrm{kg} / \mathrm{m}^{2}$ & 20.7 & 4.0 & 16.7 & 19.4 & 26.6 & 21.0 & 3.9 & 16.7 & 19.9 & 26.4 & .642 \\
\hline & FMI, $\mathrm{kg} / \mathrm{m}^{2}$ & 4.59 & 2.84 & 1.86 & 3.85 & 9.02 & 3.58 & 2.06 & 1.66 & 2.79 & 7.32 & .007 \\
\hline & FFMI, $\mathrm{kg} / \mathrm{m}^{2}$ & 16.33 & 1.91 & 14.10 & 16.17 & 19.26 & 17.40 & 2.50 & 14.69 & 16.90 & 20.15 & .001 \\
\hline & $\% \mathrm{BF}$ & 20.52 & 8.74 & 11.04 & 18.83 & 32.81 & 16.20 & 6.68 & 9.44 & 14.10 & 27.21 & .000 \\
\hline & FrI & 41.45 & 2.30 & 38.57 & 41.27 & 44.46 & 41.65 & 5.36 & 36.02 & 40.96 & 46.45 & .738 \\
\hline \multirow{7}{*}{14} & Height, $\mathrm{cm}$ & 164.4 & 9.0 & 153.5 & 166.0 & 174.5 & 167.6 & 8.3 & 157.0 & 167.0 & 179.0 & .009 \\
\hline & Weight, $\mathrm{kg}$ & 58.3 & 14.7 & 41.0 & 56.3 & 78.5 & 60.5 & 15.5 & 45.0 & 56.9 & 82.0 & .306 \\
\hline & $\mathrm{BMI}, \mathrm{kg} / \mathrm{m}^{2}$ & 21.4 & 4.3 & 16.7 & 20.5 & 28.1 & 21.4 & 4.3 & 17.1 & 20.1 & 26.9 & .945 \\
\hline & FMI, $\mathrm{kg} / \mathrm{m}^{2}$ & 4.76 & 3.30 & 1.75 & 3.56 & 9.63 & 3.60 & 2.51 & 1.48 & 2.72 & 6.95 & .009 \\
\hline & FFMI, $\mathrm{kg} / \mathrm{m}^{2}$ & 16.85 & 1.84 & 14.24 & 16.84 & 19.61 & 17.76 & 2.41 & 15.22 & 17.25 & 20.90 & .005 \\
\hline & $\% \mathrm{BF}$ & 20.31 & 9.57 & 10.17 & 17.24 & 34.02 & 15.75 & 7.44 & 8.38 & 13.43 & 26.52 & .001 \\
\hline & FrI & 41.90 & 2.67 & 38.49 & 41.79 & 45.14 & 40.16 & 4.82 & 34.79 & 39.52 & 47.05 & .001 \\
\hline \multirow{7}{*}{15} & Height, $\mathrm{cm}$ & 171.8 & 7.1 & 163.8 & 171.8 & 181.5 & 173.4 & 6.5 & 166.1 & 173.0 & 180.0 & .112 \\
\hline & Weight, kg & 63.0 & 15.5 & 48.5 & 59.0 & 84.3 & 70.3 & 15.1 & 52.2 & 68.9 & 89.9 & .001 \\
\hline & BMI, $\mathrm{kg} / \mathrm{m}^{2}$ & 21.2 & 4.2 & 17.3 & 20.1 & 27.3 & 23.3 & 4.5 & 18.7 & 22.5 & 29.5 & .001 \\
\hline & FMI, $\mathrm{kg} / \mathrm{m}^{2}$ & 4.02 & 2.78 & 1.73 & 3.03 & 7.95 & 4.32 & 2.17 & 2.06 & 3.80 & 7.09 & .403 \\
\hline & FFMI, $\mathrm{kg} / \mathrm{m}^{2}$ & 17.17 & 1.81 & 15.02 & 16.89 & 19.55 & 18.95 & 3.02 & 15.56 & 18.45 & 23.23 & .000 \\
\hline & $\% \mathrm{BF}$ & 17.65 & 7.78 & 9.97 & 15.32 & 29.30 & 17.91 & 6.36 & 10.19 & 16.88 & 24.92 & .801 \\
\hline & FrI & 41.94 & 2.24 & 38.99 & 41.67 & 44.77 & 41.45 & 5.50 & 35.88 & 40.45 & 51.08 & .429 \\
\hline \multirow{7}{*}{16} & Height, cm & 173.7 & 5.8 & 166.0 & 173.5 & 182.3 & 174.4 & 7.7 & 165.0 & 172.5 & 184.0 & .500 \\
\hline & Weight, kg & 65.6 & 12.3 & 51.6 & 63.0 & 83.3 & 69.2 & 11.2 & 59.3 & 66.4 & 84.2 & .035 \\
\hline & $\mathrm{BMI}, \mathrm{kg} / \mathrm{m}^{2}$ & 21.7 & 3.6 & 17.9 & 20.6 & 28.0 & 22.8 & 3.5 & 18.7 & 22.2 & 28.6 & .037 \\
\hline & FMI, $\mathrm{kg} / \mathrm{m}^{2}$ & 4.16 & 2.48 & 1.90 & 3.28 & 8.12 & 3.94 & 2.10 & 2.00 & 3.39 & 7.12 & .480 \\
\hline & FFMI, $\mathrm{kg} / \mathrm{m}^{2}$ & 17.55 & 1.67 & 15.67 & 17.37 & 19.78 & 18.85 & 2.15 & 16.17 & 18.81 & 21.65 & .000 \\
\hline & $\% \mathrm{BF}$ & 18.07 & 7.66 & 9.97 & 15.97 & 31.20 & 16.67 & 6.34 & 10.07 & 15.23 & 28.29 & .149 \\
\hline & FrI & 41.30 & 2.13 & 38.26 & 41.53 & 43.96 & 43.42 & 6.60 & 36.44 & 42.23 & 53.20 & .009 \\
\hline \multirow{7}{*}{17} & Height, $\mathrm{cm}$ & 175.8 & 7.0 & 167.1 & 175.2 & 185.2 & 176.4 & 7.1 & 169.0 & 177.0 & 183.1 & .582 \\
\hline & Weight, $\mathrm{kg}$ & 67.7 & 10.9 & 56.4 & 66.8 & 83.7 & 72.8 & 12.8 & 58.9 & 71.3 & 92.4 & .005 \\
\hline & $\mathrm{BMI}, \mathrm{kg} / \mathrm{m}^{2}$ & 21.9 & 3.5 & 18.4 & 21.4 & 27.3 & 23.4 & 3.9 & 18.6 & 22.5 & 29.8 & .009 \\
\hline & FMI, $\mathrm{kg} / \mathrm{m}^{2}$ & 3.73 & 2.46 & 1.33 & 3.01 & 7.63 & 3.79 & 2.39 & 1.47 & 3.12 & 8.13 & .876 \\
\hline & FFMI, $\mathrm{kg} / \mathrm{m}^{2}$ & 18.24 & 1.84 & 16.18 & 18.06 & 21.07 & 19.62 & 2.50 & 16.43 & 19.06 & 23.46 & .000 \\
\hline & $\% \mathrm{BF}$ & 15.93 & 7.75 & 7.25 & 14.06 & 26.17 & 15.38 & 7.65 & 7.09 & 13.32 & 29.19 & .651 \\
\hline & FrI & 41.17 & 2.67 & 37.92 & 41.37 & 44.66 & 42.23 & 6.43 & 35.44 & 41.05 & 51.32 & .192 \\
\hline
\end{tabular}

Note: Mean - average mean values; SD - standard deviation; P10 -10 ${ }^{\text {th }}$ percentile; P50 - $50^{\text {th }}$ percentile; P90 - $90^{\text {th }}$ percentile; BMI-Body Mass Index; FMI-Fat Mass Index; FFMI-Fat-Free Mass Index; \% BF-percentage of Body Fat; FrI-Frame-Index; $\mathrm{p}$ - level of significance

The average height of 7-year-old boys is $128.0 \pm 5.8$. After this period the values of height increase to $176.4 \pm 7.1 \mathrm{~cm}$ for 17 -year-old boys. They are slightly taller than their peers 10 years ago. The differences with boys are significant from their11 to 14 years of age, and with girls up to the age of $10(\mathrm{p}<0.05)$. The mean value of weight increase from $28.4 \pm 6.3 \mathrm{~kg}$ at 7 years of age to $72.8 \pm 12.8 \mathrm{~kg}$ for 17 year-old boys, respectively. 
The average values of the Body Mass Index (BMI) increase from $17.2 \pm 6.3 \mathrm{~kg} / \mathrm{m}^{2}$ for 7 year-olds to $23.4 \pm 3.9 \mathrm{~kg} / \mathrm{m}^{2}$ for 17 year-olds, respectively. The differences between BMI of the boys from the two samples are significant at $11,15,16$ and 17 years of age $(p<0.05)$.

The average value of the Fat Mass Index (FMI) of 7-year-old boys is $2.86 \pm 1.65 \mathrm{~kg} / \mathrm{m}^{2}$ and it increases to $3.79 \pm 2.39 \mathrm{~kg} / \mathrm{m}^{2}$ in 17 -year-olds. The results show that only $13-14$-year-old contemporary boys have significantly higher values of their FMI compared with their coevals in $1998(\mathrm{p}<0.05)$.

The Fat-Free Mass Index (FFMI) of boys also increases from $14.32 \mathrm{~kg} / \mathrm{m}^{2} \pm 1.57$ $\mathrm{kg} / \mathrm{m}^{2}$ at 7 years of age to $17.25 \pm .59 \mathrm{~kg} / \mathrm{m}^{2}$ at 17 years of age. The differences between the average values of FFMI of boys from the two samples are significant above the age of 9 years to the end of the period $(\mathrm{p}<0.05)$ and are higher for contemporary boys.

The average Body Fat $(\% \mathrm{BF})$ percentage for 7 year-old boys is $15.86 \% \pm 6.74 \%$. Its values increase until 10 years of age up to $19.64 \% \pm 8.07 \%$, subsequently decreasing to 15.38 $\% \pm 7.65 \%$ for 17 - year-old boys. Between 7-8 years of age and 12-14 years of age the average percentage of $\mathrm{BF}$ for contemporary boys is significantly lower compared with \% BF of their coevals in $1998(\mathrm{p}<0.05)$. After this period the values of \% BF of the two samples of boys are very similar.

The average values of the Frame-Index for boys also increase with the age from $39.39 \pm 3.65$ UI for 7-year-old boys to $42.23 \pm 6.43$ UI for 17 year-old-boys. But the contemporary 7-10 year-old boys have significantly lower values of the Frame-Index, compared with their coevals. After this period the values of this index are very similar for the two samples of boys, considering them up to 14 years of age. The summarized results show a significant decrease in the index values in almost all age groups and both sexes for the past 10 years with the exception of 11- and 16-year-old boys and 17-year-old girls.

Figures 1 and 2 present the graphic curves of percentiles 3rd, 10th, 50th, 90th and 97th of the Frame-Index for Plovdiv children and adolescents belonging to both sexes and both samples. It is noteworthy that the skeletal robustness does not decrease with time in all percentile groups. The values of the 90th and 97th percentiles are higher for children nowadays from all age groups, while the values of the 10th and 3rd percentiles of the index are lower. The differences in the values of 10th percentile measured in the course of ten years ranged between 0.8 and 5.3 index units for boys, and from 2.3 to 6.9 index units for girls. 
Figure 1. Percentiles of skeletal robustness, assessed by the Frame index (7-17- year-old Plovdiv girls, 1998 and 2008)

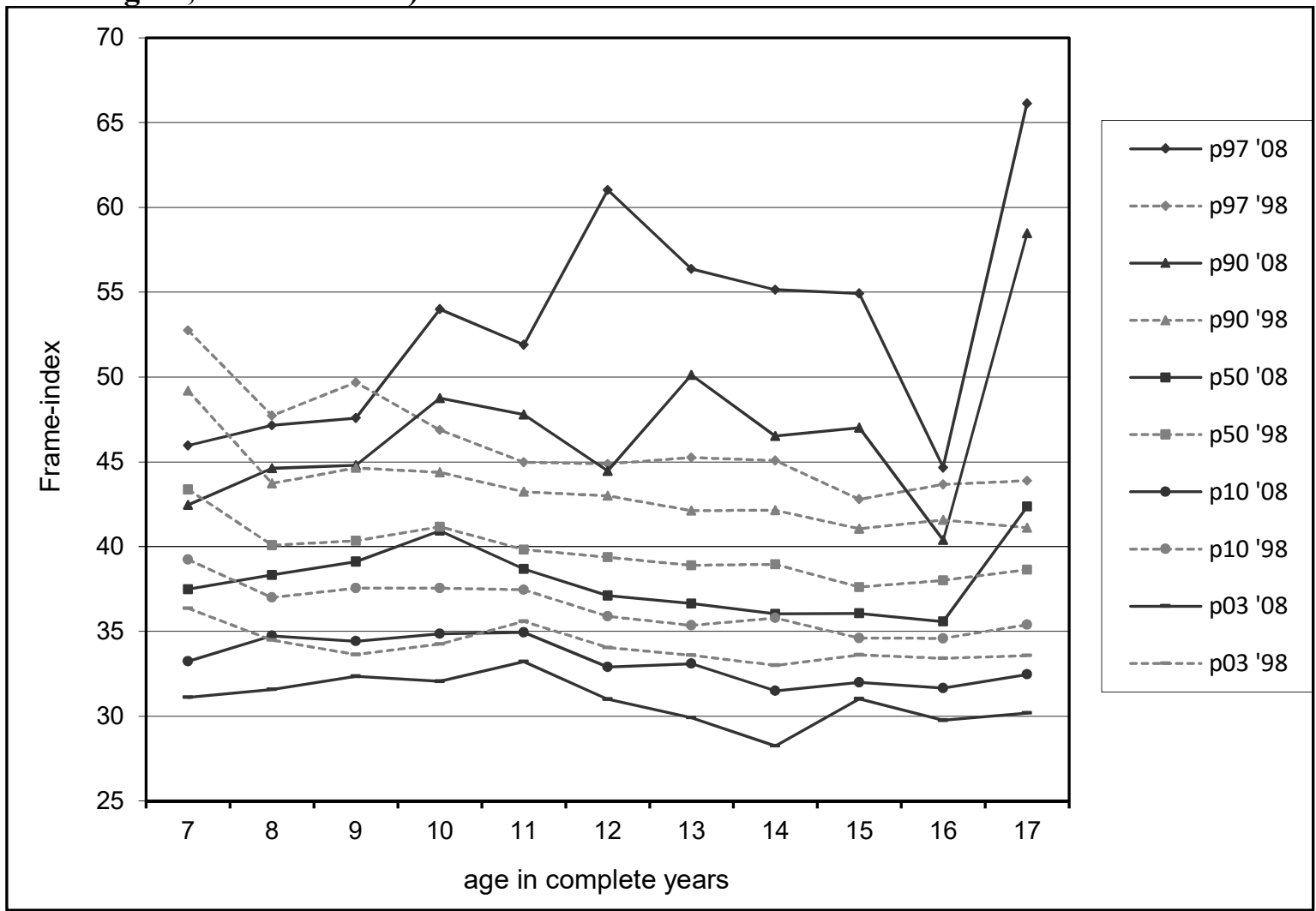

Figure 2. Percentiles of skeletal robustness, assessed by the Frame index (7-17- year-old Plovdiv boys, 1998 and2008

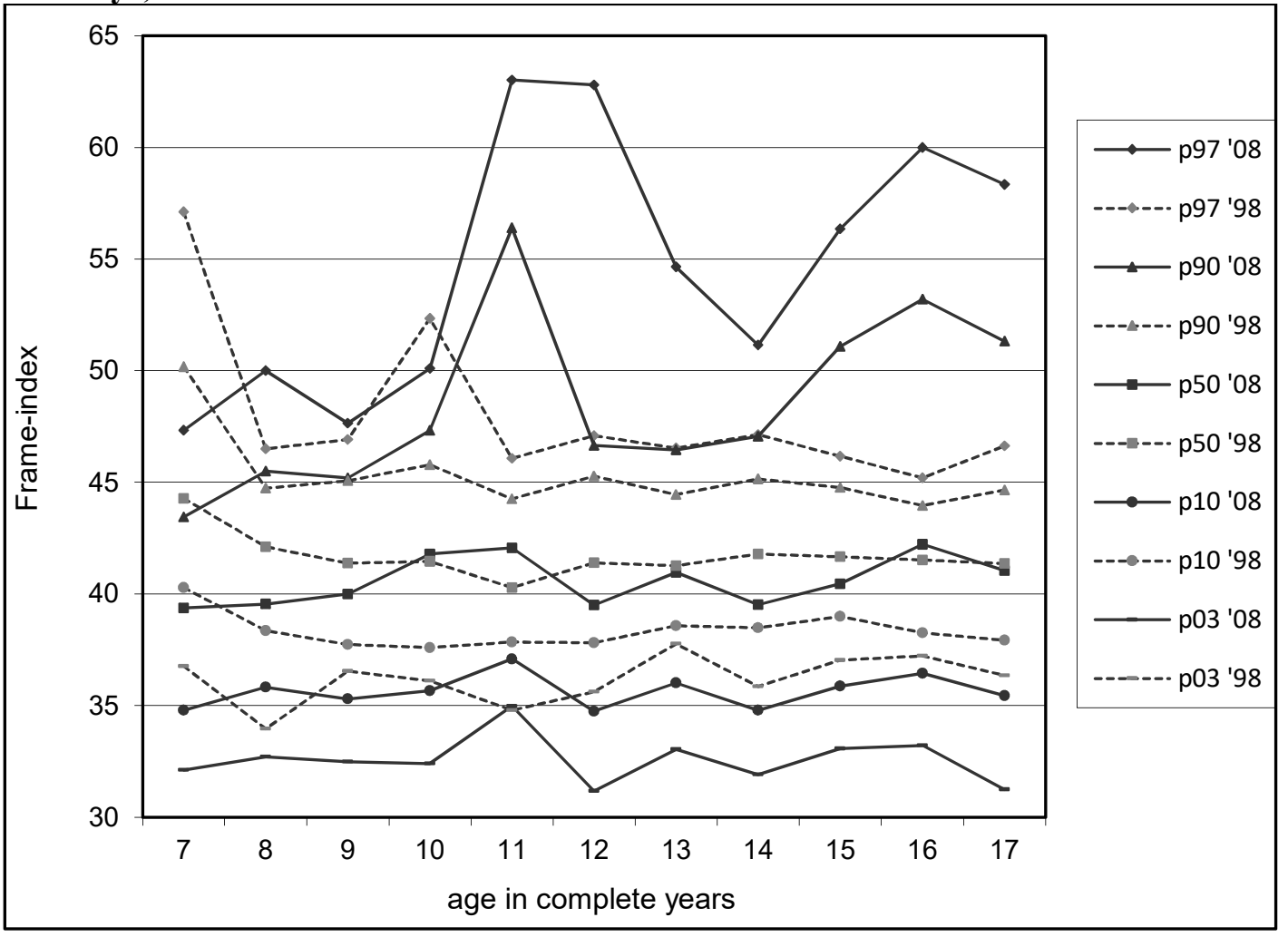


Table 3 presents the relationship between the Frame-Index i.e. external skeletal robustness and the percentage of Body Fat and the Body Mass Index. The analysis shows that there are no significant correlations between the three body components for both sexes.

Table 3. Coefficients of the correlation between the Frame-Index and BMI, respectively Frame-Index and \% BF

\begin{tabular}{|c|c|c|c|c|c|}
\hline \multirow{2}{*}{ Girls } & \multicolumn{2}{|c|}{ r } & \multirow{2}{*}{ Boys } & \multicolumn{2}{|c|}{ R } \\
\cline { 6 - 7 } & BMI & \%BF & & BMI & \%BF \\
\hline 7 y. & 0.329 & 0.348 & 7 y. & 0.237 & 0.337 \\
\hline 8 y. & 0.329 & 0.274 & 8 y. & 0.474 & 0.450 \\
\hline 9 y. & 0.421 & 0.403 & 9 y. & 0.527 & 0.463 \\
\hline 10 y. & 0.330 & 0.170 & 10 y. & 0.382 & 0.472 \\
\hline 11 y. & 0.353 & 0.135 & 11 y. & 0.263 & -0.025 \\
\hline 12 y. & 0.052 & -0.102 & 12 y. & 0.310 & 0.182 \\
\hline 13 y. & 0.251 & -0.098 & 13 y. & 0.285 & 0.157 \\
\hline 14 y. & 0.234 & 0.048 & 14 y. & 0.281 & 0.128 \\
\hline 15 y. & 0.244 & -0.103 & 15 y. & 0.447 & 0.188 \\
\hline 16 y. & 0.317 & 0.085 & 16 y. & 0.247 & 0.139 \\
\hline 17 y. & 0.358 & -0.335 & 17 y. & 0.328 & 0.153 \\
\hline
\end{tabular}

Note: r-correlation coefficient; BMI- Body Mass Index; \% BF-percentage of Body Fat

\section{Discussions}

Body components depend on height and weight. Our results of height show that children nowadays have normal growth. They are slightly taller compared with their peers 10 years ago. Age differences in body weight relate to the changes in height, and it is important to consider the ratio of weight to height when comparing the two samples.

The Body Mass Index (BMI) gives such information, not considering the Fat-Free Mass Index (FFMI) and the Fat Mass Index (FMI). Our results show that boys have higher values of FFMI throughout the whole age period of examination, while girls have higher values of $\% \mathrm{BF}$ and FMI between 12 and17 years of age. This fact occurs in both samples - in 1998 and 2008. For children and adolescents in 2008, however, the fat-free mass component is higher, and the fat component is lower than this of their peers 10 years ago. The changes of the two components of the BMI such as FMI and FFMI in Bulgarian children and adolescents from the city of Sofia were published by Mitova (2009).

The important health problem concerning skeletal robustness was discussed in previous studies by Scheffler (2011) as far as 6-to 12-year-old German children are concerned and by Rietsch, Godina and Scheffler (2013) as far as 6-10-year-old Russian and German children are concerned. One of the newest studies devoted to this problem is by Mumm et al. (2018) observing children from different European countries (Germany, Poland, Czech Republic, Russia), India and South Africa. The study presents the new European references for external skeletal robustness from birth to adulthood and their international comparisons.

Our study referring to the first period of time presents the data of the external skeletal robustness for Bulgarian children, based on the Frame-Index. Our results demonstrate the changes that have occurred during the 10-year period. The summarized results show a significant decrease of the index values for almost all age groups and both sexes for the past 10 years with the exceptions of 11 - and 16-year-old boys and 17-year-old girls. The results point at the fact 
that skeletal robustness does not decrease in time in all percentile groups. The values of the $90^{\text {th }}$ and $97^{\text {th }}$ percentiles are higher for contemporary children belonging to all age groups, while the values of the $10^{\text {th }}$ and $3^{\text {rd }}$ percentiles of the index are lower. The differences in the values of $10^{\text {th }}$ percentile measured in the course of 10 years ranged between 0.8 and 5.3 index points for boys, and from 2.3 to 6.9 index points for girls. These results correspond to a lower skeletal robustness from $2 \%$ to $15.7 \%$ for boys, respectively from $7 \%$ to $18 \%$ for girls. It is noteworthy that the values of the $10^{\text {th }}$ percentile in contemporary 10-17-year-old girls and 11-17-year-old boys are lower than the $3^{\text {rd }}$ percentile of the children examined in 1998-1999. This is worrying because reduced skeletal mass and skeletal robustness are risk factors for osteoporosis in later years of an individual's life (Langenbeck, 2005; Sheffler, 2011; Rietsch, Godina and Scheffler, 2013; Mumm et al., 2018).

Our results also present how the skeletal robustness is associated with the \% $\mathrm{BF}$ and the BMI. The results of the correlation analysis show that there are no significant correlations between the three body components. The fact that there is no correlation between the skeletal robustness and the body fat percentage means that it is not necessary for a child with a high percentage of body fat to have a low value of the Frame-Index. This makes the interpretation of results concerning external skeletal robustness very complicated, especially in the low percentile groups. In general, skeletal mass depends on genetic factors in $60-80 \%$ and in $20-40 \%$ it is modified by external factors, including nutrition and physical activity (Cameron and Demerath, 2002). Over the past 10 years, no genetic changes have occurred in Plovdiv population and the lower percentile values of the Frame-Index are likely to be a result of the influence of external factors that are probably connected with reduced physical activity. Our results confirm the data of a previous study by Sheffler (2011) and Rietsch, Godina and Scheffler (2013) showing a decrease in external skeletal robustness of contemporary German and Russian children, connected by the authors with an increase in values of BMI and body fat percentage, as well with a decrease in physical activity being the most important reason for shrinking skeletal robustness.

The latter leads to an increase in the number of overweight individuals, who have a higher percentage of body fat, but not lower values of the Frame-index. The different development of body components is related to two phenomena. On the one hand, under the same environmental conditions and depending on the genetic constitution of the body, there are gracile and robust individuals in both samples. On the other hand, the individuals registered with overweight and obesity should develop their own muscles and bones to maintain their body, which is probably due to the increased skeletal robustness. A harmonious and healthy somatic and psychic development can be ensured by a nutritional balance, adapted according to age, occupation, and the environment in which an individual lives (Baciu, 2014).

\section{Conclusions}

While growing, contemporary children accumulate more fat -free body mass per unit of height while their peers a decade ago accumulated more fat tissue per unit of height.

The increase in the percentage of body fat and the values of the Fat-Mass Index of body composition and the decrease in the Frame-Index i.e. of the external skeletal robustness for contemporary children and adolescents from Plovdiv were entirely or partially caused, by reduced physical activity. In this context physical activity should be particularly investigated.

\section{Bibliography}

1. Baciu, A., 2011. Anthropological-medical aspects of feeding behavior of children in modern society. Review of Global Medicine and Healthcare Research (RGMHR), 2 (1), pp. 79-99. 
2. Baciu, A., 2013. Aspects anthropologique et médicaux des campagnes pour une alimentation saine chez lez jeunes et adolescents - Anthropological and medical aspects of the campaigns for a healthy dietary among young people and teenagers. Biométrie Humaine et Anthropologie, 31, (3-4), pp. 91-98.

3. Baciu, A., 2014. Alimentaţia tinerilor în condiţiile actuale din perspectivă antropo-medicală/ Teenage alimentation under the current conditions from an antropo-medical perspective. In „Educaţia nutriţională şi provocările lumii contemporane”. București: Colegiul Economic „Viilor”.

4. Basterfield, L., Adamson, A.J., Frary, J.K., Parkinson, K.N., Pearce, M.S. and Reilly, J.J.; Gateshead Millennium Study Core Team, 2011. Longitudinal Study of Physical Activity and Sedentary Behavior in Children. Pediatrics, 127(1), pp. 24-30.

5. Cameron, N. and Demerath, E., 2002. Critical Periods in Human Growth and Their Relationship to Disease of Aging. Physic. Anthropol. Rev., 45, pp. 159-184.

6. Chtecov, V., 1970. Body composition of human, Antrhopology 1969. Moscow: Soviet Union Academy of Sciences Publisher, pp. 1-128 (Article in Russian).

7. Daniels, S., Khoury, P. and Morrison, J., 1997. The utility of the body mass index as a measure of body fatness in children and adolescents: Differences by race and gender. Pediatrics, 99 (6), pp. 804-807.

8. Deurenberg P., Weststrate J., and Seidell, J., 1991. The body mass index as a measure of body fatness: age - and sex - specific prediction formulas. Br J Nutr., 65, pp. 105-114.

9. Erbersdobler, H., Heseker, H. and Wolfram, G., 2005. Adipositas - Eine Herausforderung für's Leben. Stuttgart: Wissenschaftliche Verlagsgesellschaft mbH Heseker. mbH.

10. Freedman, D., Khan, L., Serdula, M., Dietz, W., Srinivasan, S. and Berenson, G., 2005. The relation of childhood BMI to adult adiposity: the Bogalusa Heart Study. Pediatrics, 115(1), pp. 22-27.

11. Frisancho, A., 1984. New standards of weight and body composition by frame size and height for assessment of nutritional status of adults and elderly. Am. Journ. of Clinical Nutrition, 40, pp. 808-819.

12. Frisancho, A., 1990. Anthropometric standards for the assessment of growth and nutritional status. Ann Arbor., University of Michigan Press, p. 28.

13. Klimatskaya, L., Laskiene, S. and Shpakou, A., 2011. Lifestyle and health behaviour of school aged children in Krasnoyarsk (Russia), Lithuania and Grodno (Belarus). Prog. Health Sci., 1(2), pp. 39-45.

14. Langenbeck, U., 2005. Erb- und Umweltfaktoren in der Entstehung der Osteoporose: Wege zu Prädiktion und Prävention. Dtsch. Arztebl., 102 (10), pp. 664, 555, 521.

15. Lömmle, L., Worth, A., and Bös, K., 2012. Socio-demographic correlates of physical activity and physical fitness in German children and adolescents. Eur J Public Health. [ejournal] 22(6), pp. 880 - 884. https://doi.org/10.1093/eurpub/ckr191.

16. Martin, R. and Saller, K., 1957. Lehrbuch der Anthropologie. Stuttgart: Gustav Fischer Verlag.

17. Mitova, Z., 2009. Anthropological characteristics of physical development, body composition and body nutritional status of 9-15-year-old children from Sofia. PhD-thesis, Sofia, BAS. (Article in Bulgarian).

18. Mladenova, S. and Nikolova, M., 2005. Components of body mass and their relations during the growth period of the boys. Proceedings of the Balkan Scientific Conference of Biology, Plovdiv, pp. 138-149.

19. Mladenova, S. and Kodgebasheva, D., 2010. Changes in Components of Body Mass and Their Relation During Period of Growth in Girls from Smolyan Region, Bulgaria (Anthropometric Study). Biotechnology \& Biotechnological Equipment, [e-journal] 24:sup1, pp. 347-353. https://doi.org/10.1080/13102818.2010.10817860. 
20. Mumm, R., Godina, E., Koziel, S., Musalek, M., Sedlak, P., Wittwer-Backofen, U., Hesse, V., Dasgupta, P., Henneberg, M. and Scheffler, C., 2018. External skeletal robusticity of children and adolescents - European references from birth to adulthood and international comparisons. Anthropol. Anz., 74(5 Supplement), pp. 383-391.

21. Pietrobelli, A., Faith, M., Allison, D., Gallagher, D., Chiumello, G. and Heymsfield, S., 1998. Body mass index as a measure of adiposity among children and adolescent: a validation study. Journal of Pediatrics, 132, pp.204-210.

22. Popkin, B.M., Adair, L.S. and Ng, S.W., 2012. Global nutrition transition and the pandemic of obesity in developing countries. Nutrition reviews, 70(1), pp. 3-21.

23. Laskiene, K., Godina, E. and Scheffler, C., 2013. Decreased External Skeletal Robustness in Schoolchildren - A Global Trend? Ten Year Comparison of Russian and German Data. PLoS ONE, [e-journal] 8(7), p. 68195. https://doi.org/10.1371/journal.pone.0068195

24. Rolland-Cachera, M., 1993. Body composition during adolescence: methods, limitations and determinants. Hormonal Research, 39, pp. 25-40.

25. Scheffler, C., 2011. The change of skeletal robustness of 6-12 years old children in Brandenburg (Germany) - Comparison of body composition 1999-2009. Anthrop. Anz., 68(2), pp. 153-165.

26. Slaughter, M., Lohmann, T., Boileau, R., Horswill, C., Stillmann, R., Van Loan, M. And Bemben, D., 1988. Skinfold equations for estimation of body fatness in children and youth. Hum. Biol., 60, pp.709-723.

27. Verloigne, M., Van Lippevelde, W., Maes, L., Yıldırım, M., Chinapaw, M., Manios, Y., Androutsos, O., Kovács, E., Bringolf-Isler, B., Brug, J., ... and De Bourdeaudhuij, I., 2012. Levels of physical activity and sedentary time among 10- to 12-year-old boys and girls across 5 European countries using accelerometers: an observational study within the ENERGY-project. The International Journal of Behavioral Nutrition and Pysical Activity, [e-journal] 9, p. 34. https://doi.org/10.1186/1479-5868-9-34.

28. $* * *$ World Medical Association Declaration of Helsinki, 2000. Ethical principles for medical research involving human subjects. JAMA, 284(23), pp. 3043-3045. 